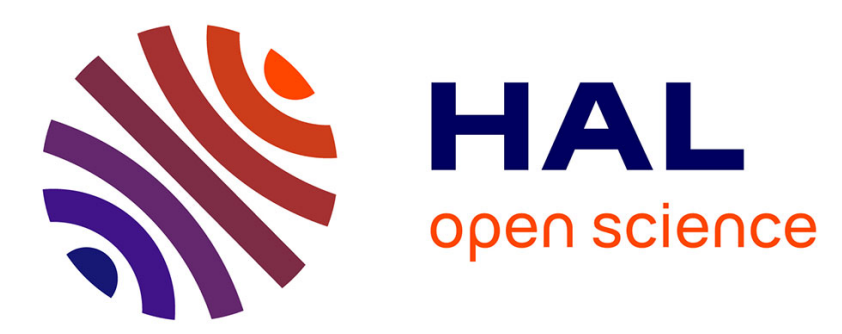

\title{
The effects of the finite spatial resolution of thermoplastic recording materials on the holographic image \\ István Bányász
}

\section{- To cite this version:}

István Bányász. The effects of the finite spatial resolution of thermoplastic recording materials on the holographic image. Journal de Physique III, 1993, 3 (7), pp.1435-1444. 10.1051/jp3:1993210 . jpa-00249009

\section{HAL Id: jpa-00249009 https://hal.science/jpa-00249009}

Submitted on 1 Jan 1993

HAL is a multi-disciplinary open access archive for the deposit and dissemination of scientific research documents, whether they are published or not. The documents may come from teaching and research institutions in France or abroad, or from public or private research centers.
L'archive ouverte pluridisciplinaire HAL, est destinée au dépôt et à la diffusion de documents scientifiques de niveau recherche, publiés ou non, émanant des établissements d'enseignement et de recherche français ou étrangers, des laboratoires publics ou privés. 
Classification

Physics Abstracts

$42.40 \mathrm{D}-42.40 \mathrm{~F}-42.40 \mathrm{H}$

\title{
The effects of the finite spatial resolution of thermoplastic recording materials on the holographic image
}

\author{
István Bányász \\ Institute of Isotopes of the Hungarian Academy of Sciences, Budapest, P.O.B. 77, H-1525 \\ Hungary
}

(Received 20 November 1992, accepted 21 April 1993)

\begin{abstract}
The first exact method for the evaluation of the effects of the finite, band-limited spatial resolution of the thermoplastic - photoconductor recording materials on the reconstructed holographic image is presented in this paper. This new method is based on substitution of the amplitude modulation transfer function (i.e. the square root of the MTF) of the recording material into the double Fresnel-Kirchhoff integral describing the complex amplitude of the reconstructed image. The contrast of the reconstructed image as a function of various recording parameters such as the centre and width of the amplitude modulation transfer function of the recording material, the object position and the reference beam angle have been computed. Numerical calculations have been carried out for thin holograms of microline objects in two recording geometries, one with a tilted object plane.
\end{abstract}

\section{Introduction.}

Thermoplastic holographic recording materials have been extensively studied both theoretically and experimentally since the 1960's. It was Glenn [1] who first reported the use of thermoplastic materials for high density recording of electrical signals by electron beam writing. The first holographic recording in an organic photoconductor coated with an insulating thermoplastic was made by Urbach and Meier [2]. Later on a number of different versions of these devices has been developed. The Lumatron [3] was a modified version of Glenn's original direct electron beam writing method applied to high resolution optical storage and projection display devices. The Ruticon family of reusable recording devices was developed by Sheridon [4]. These devices differed from those of Urbach and Meier mainly in the use of different materials (elastomers) and in the method of the electric charging of the device.

A number of papers appeared on the various experimental characteristics (e. g. spectral sensitivity, MTF) of the thermoplastics [5-10]. Since the holographic recording process in thermoplastic photoconductor devices involves several steps besides the photochemical reaction (charging, heating), and the devices studied were usually non standard ones, there is a vast diversity in the reported characteristics. As a consequence, it is difficult to compare the results of different authors. 
Though it is not easy to establish general laws describing the properties of thermoplastic recording materials, several theoretical papers on their physical properties can be found in the literature [11-15]. In some of these works theoretical relationships between some physical properties and the spatial frequency of the periodic surface relief pattern of the thermoplastic have been established. Budd [12] calculated the normalized growth rate of the surface deformations as a function of the product of the thickness of the layer and the magnitude of the grating vector. If it is assumed that the diffraction efficiency is a monotonic function of this growth rate then both of them should have their maxima at the same spatial frequency (for a fixed layer thickness). It was the author of the present paper who made this assumption, and by comparing the results of Budd by the experimental MTF curves reported by Lo et al. [9], he found that Budd's theory predicts a centre frequency of 595 lines/mm for the MTF of a thermoplastic of the thickness of $0.8 \mu \mathrm{m}$ while the experimental MTF has its peak at 520 lines $/ \mathrm{mm}$ as it can be seen in figure 1. Some theoretical considerations concerning the holographic characteristics of thermoplastics can be found in the report of Stewart et al. [7], too. They calculated the variations of the electric potential on the surface of the thermoplastic using an equivalent electric circuit model.

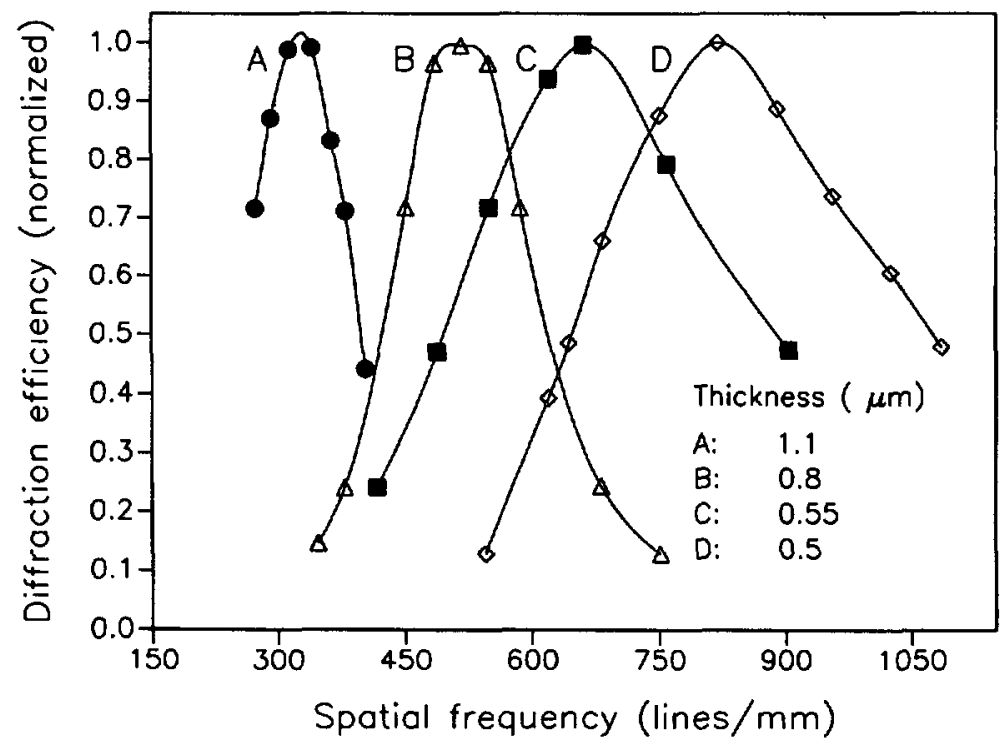

Fig. 1. - Diffraction efficiency v's. spatial frequency with thermoplastic film thickness as parameter, after Lo et al. [9].

A concise review of the properties of holographic recording materials is the paper of Urbach an Meier [16]. Holography with thermoplastics has been thoroughly treated in the review of Urbach [17]. In the conclusions of his review he points out the advantages of holograms recorded in thermoplastic coated photoconductor devices, namely their relatively high diffraction efficiency (over $30 \%$ ), the attainable high resolution limit (up to $4000 \mathrm{lines} / \mathrm{mm}$ ) and high useful bandwidth (in excess of 1000 lines/mm in some devices), the suppression of intermodulation distortions as a result of this band-pass spatial frequency response, and high sensitivity (comparable to that of the silver halide materials). 
Based on the statements of the previous paragraphs, one could think that thermoplastic photoconductor devices are excellent candidates for reconfigurable holographic optical elements even when high resolution and field range is required. However, at least to the author's knowledge, no study concerning the ultimate capabilities in image resolution of these recording media has been appeared so far.

The aim of the present work was to develop an exact method for the evaluation of the direct effects of the band-limited MTF of the thermoplastic materials on the reconstructed image of an arbitrary object retrieved from an arbitrary hologram. The following calculations follow a similar principle to those presented in some papers of the author [18-20].

Relationships between the contrast of the reconstructed image and various parameters of the holograms have been established. The following calculations apply to one dimensional thin amplitude and shallow phase relief holograms.

\section{Evaluation of the effects of the MTF on the reconstructed image.}

1.1 THE RECORDING GEOMETRIES AND THE OBJECT. - One dimensional holograms of one dimensional microline objects have been studied in the present work. The parameters of the two recording geometries are shown in table I. The coordinate along the hologram is denoted by $\xi$. The coordinate in the object plane (or more precisely the object line) is denoted by $u$ and that line is inclined at an angle $\alpha_{0}$ to the hologram line. The object-to-hologram distance is defined by $R_{0}$, the separation of the hologram centre and the object centre. The angle of incidence of the plane reference wave is $\alpha_{r}$. The hologram width is $L$. The edges of the hologram are $\xi_{1}$ and $\xi_{2}$. The hologram is reconstructed by a plane wave which is the exact conjugate of the reference wave.

Table I. - The parameters of the recording geometries.

\begin{tabular}{|c|c|c|c|c|c|c|}
\hline Geometry & $\mathbf{R}_{n}(\mathrm{~mm})$ & $\mathbf{L}(\mathrm{mm})$ & $\xi_{1}$ & $\alpha_{n}\left(\mathbf{d e g}_{\text {. }}\right)$ & $\left.\alpha_{\text {(deg. }}\right)$ & $\mathbf{N}$. A. \\
\hline \hline $\mathbf{E}$ & 32 & 84 & -42.0 & 0.0 & -40.0 & 0.795 \\
\hline $\mathbf{F}$ & 32 & 84 & -42.0 & 20.0 & -20.0 & 0.800 \\
\hline
\end{tabular}

The object was a five-element Ronchi-ruling of a grating constant of $2 \mu \mathrm{m}$. In coherent imaging the evaluation of the reconstructed images of such objects gives a more realistic measure of the quality of the holograms than that of one- or two-point objects.

The wavelength of the light was $632.8 \mathrm{~nm}$ for all the calculations presented in the paper. Note that the numeric apertures of the two recording geometries are high and practically of the same value. This fact on the one hand ensures a good resolution when the image degrading factors are not in effect, on the other hand it makes the paraxial approximations inadequate.

1.2 Evaluation OF THE RECONSTRUCTED IMAGE, - As it was stated before, all the subsequent calculations are based on the numerical evaluation of the Fresnel-Kirchhoff integral. For the case of the present geometry, the diffraction-limited complex amplitude distribution of the reconstructed image is the following [18] :

$$
\underline{V}(x, z)=\int_{\xi_{1}}^{\xi_{2}} \int_{u_{1}}^{u_{2}} \underline{U}(u) \frac{\cos \theta \cos \rho}{r_{1} r_{2}} \exp \left(i k\left(r_{1}-r_{2}\right)\right) \mathrm{d} u \mathrm{~d} \xi
$$


where $\underline{U}(u)$ is the complex amplitude transmittance of the object, $\theta$ and $\rho$ are the inclination angles for the diffraction at the object and hologram plane, respectively, $r_{1}$ and $r_{2}$ are the separations of the source and observation points at the first and the second diffraction. It is assumed that there is no wavelength change at reconstruction.

Evaluating the above formula for a special holographic geometry, one obtains a diffraction limited image of the object.

Though the holograms recorded in thermoplastic materials are surface relief gratings and consequently they modify the phase of the reconstruction wave, the concept of the MTF and amplitude modulation transfer function (AMTF) [19-21] is still applicable. The MTF of a thermoplastic material is considerably affected by its thickness (its peak is shifted towards the higher frequencies for decreasing thickness, the development time etc. (See for example [9].) The MTF of these materials is usually asymmetrical, but in certain cases even a symmetrical one like a Gaussian function gives a good fit. A typical series of experimental MTF curves can be seen in figure 1. A fit to curve $\mathbf{B}$ of that figure with a Gaussian function (made by the author) is shown in figure 2. It can be seen that choosing this kind of model function does not introduce significant deviations from the experimental curve. On the other hand, this choice facilitates numerical calculation. Let us assume that the AMTF of the material is of the Gaussian form :

$$
\sigma(\nu)=\exp \left(-\left(\frac{\nu-\nu_{0}}{w_{0}}\right)^{2}\right)
$$

where $\nu_{0}$ and $w_{0}$ are the centre and the width of the AMTF.

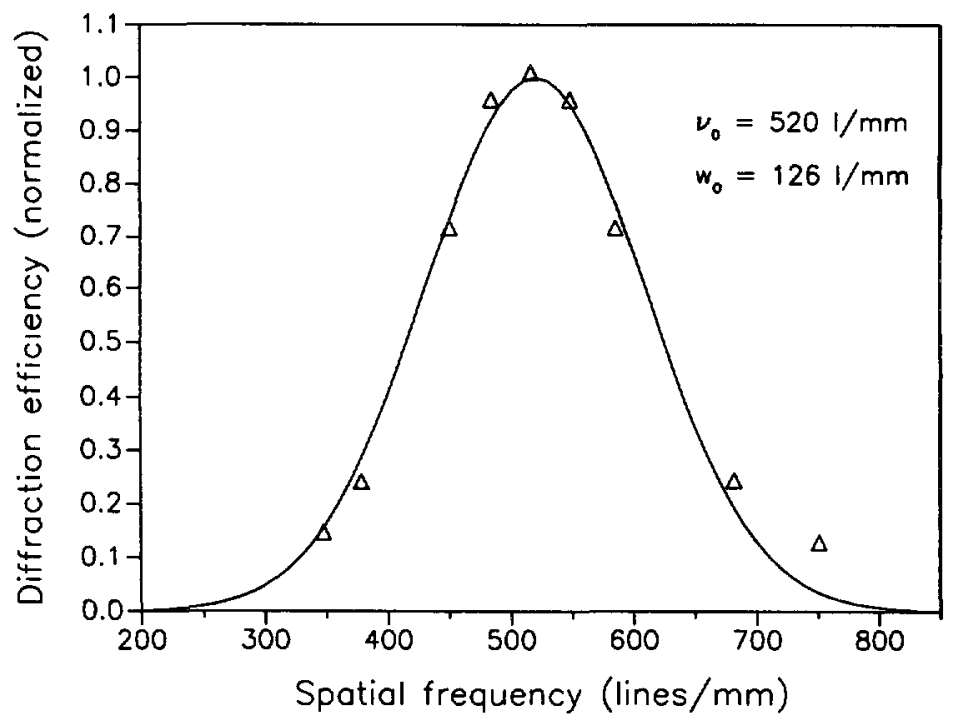

Fig. 2. - A Gaussian function fit of curve B of figure 1. The parameters are indicated in the figure.

Equation (2) is valid for holograms recorded with plane waves. However, in holograms of objects having some spatial structure the spatial frequency of the grating, $\nu$ is not constant but varies with the position $(\xi)$. In off-axis holography the spatial frequency variations through the holographic grating are mainly determined by the angle of incidence of the reference wave and 
the position of the object $[20,21]$. In our case the object is very small compared to the hologram width and to the object-hologram separation. Furthermore, the intensity distribution of the object is a slowly varying function. As a consequence, any sufficiently small part of the hologram can be regarded as an elementary plane wave hologram and it can be assigned a welldefined grating frequency of the following form to

$$
\nu(\xi)=\frac{\sin \beta_{0}(\xi)-\sin \alpha_{\mathrm{r}}}{\lambda}
$$

where $\xi$ is the coordinate along the hologram, $\beta_{0}(\xi)$ is the local angle of incidence of the central object ray, $\alpha_{\mathrm{r}}$ the (constant) angle of incidence of the reference wave and $\lambda$ is the wavelength of the light.

Knowing the spatial frequency at each point of the hologram, one can easily obtain the contribution of the elementary hologram centred at that point to the amplitude of the holographic image by multiplying the diffraction limited amplitude of the reconstructed image with the value of the amplitude modulation transfer function at that spatial frequency. Consequently, the insertion of $\sigma(\nu)$ into (1) yields the following formula for the complex amplitude of the reconstructed image :

$$
\underline{V}(x, z)=\int_{\xi_{1}}^{\xi_{2}} \int_{u_{1}}^{u_{2}} \underline{U}(u) \frac{\cos \theta \cos \rho}{r_{1} r_{2}} \exp \left(-\left(\frac{\nu-\nu_{0}}{w_{0}}\right)^{2}\right) \exp \left(i k\left(r_{1}-r_{2}\right)\right) \mathrm{d} u \mathrm{~d} \xi .
$$

\section{Results.}

Reconstructed images of the test object have been evaluated for a range of realistic values of both parameters of the Gaussian AMTF as well as for a range of the reference wave angle, $\alpha_{\mathrm{r}}$ in both geometries. In order to get a quantitative picture of the MTF effects, the contrast of the reconstructed image, defined as the integral of the intensity distribution of the reconstructed image over the transparent object lines divided by that over the opaque lines (including two opaque lines at both ends) has been calculated.

The contrast of the reconstructed image as a function of the width of the Gaussian AMTF function in geometries $\mathrm{E}$ and $\mathrm{F}$ is presented in figures 3 and 4 . The parameter is

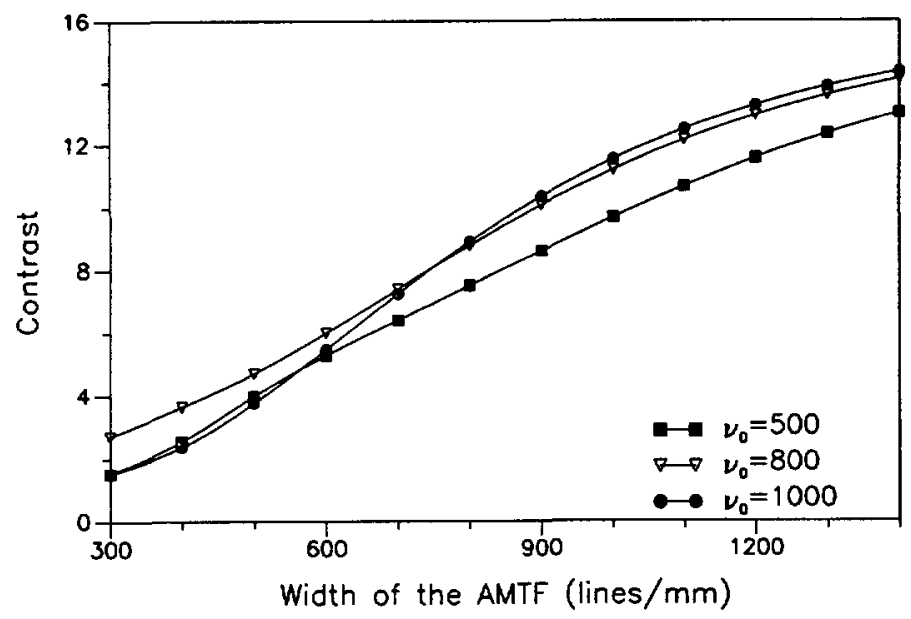

Fig. 3. - Contrast of the reconstructed image i's. the width of the Gaussian AMTF in geometry E. 


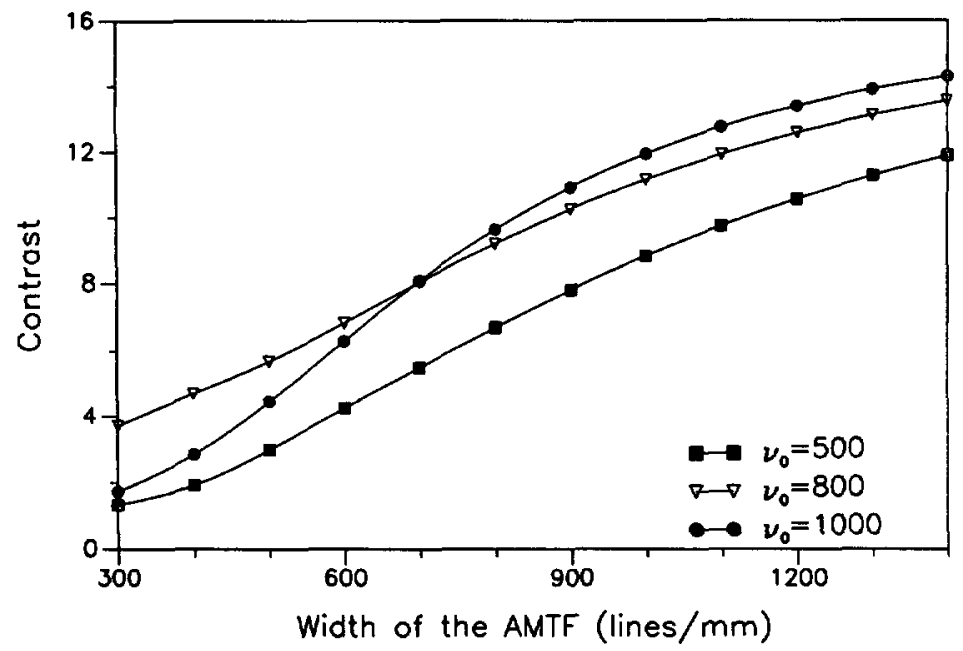

Fig. 4. - Contrast of the reconstructed image $v$ 's. the width of the Gaussian AMTF in geometry F.

$\nu_{0}$, the centre of the AMTF. It is the curves corresponding to the lowest value of $\nu_{0}(500$ lines $/ \mathrm{mm})$ which have the lowest values of contrast through the whole range of widths (except the $w_{0}<600 \mathrm{l} / \mathrm{mm}$ part of Fig. 3). The AMTFs centred at $800 \mathrm{l} / \mathrm{mm}$ give better contrasts than those centred at $1000 \mathrm{l} / \mathrm{mm}$ for widths smaller than $700 \mathrm{l} / \mathrm{mm}$, but the AMTFs centred at $1000 \mathrm{l} / \mathrm{mm}$ give higher contrasts in the $w_{0}>700 \mathrm{l} / \mathrm{mm}$ region in both geometries. The intensity distribution of the reconstructed image in geometry $\mathrm{E}$, in case of AMTFs of different width but centred at the same spatial frequency are shown in figure 5.
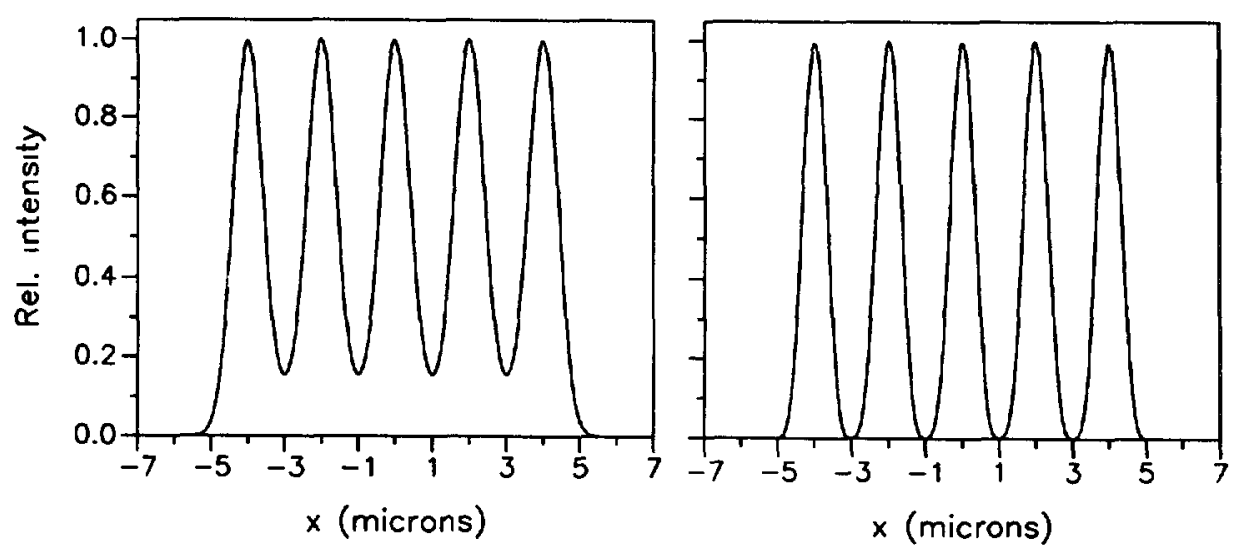

Fig. 5. - Intensity distribution of the reconstructed image in geometry E, with $\nu_{0}=1000 \mathrm{l} / \mathrm{mm}$. Left : $w_{0}=500 \mathrm{l} / \mathrm{mm}$, right : $w_{0}=1000 \mathrm{l} / \mathrm{mm}$.

As a complementary representation of the same function of two variables, the contrast of the reconstructed image as a function of the centre of the Gaussian AMTF $\left(\nu_{0}\right)$ at two values of $w_{0}$ are shown in figures 6 and 7 . Note the occurrence of two maxima in both geometries at $w_{0}=400 \mathrm{l} / \mathrm{mm}$, with a peak-to-valley ratio of about 2 . This fact implies that the centre of the 


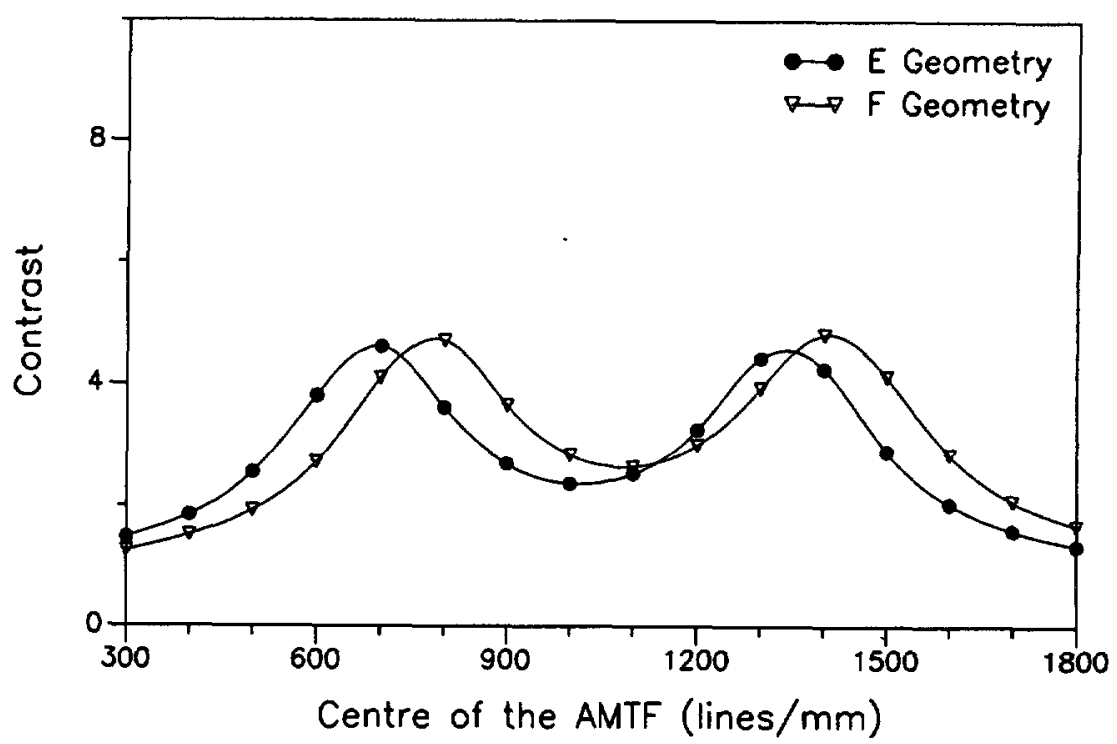

Fig. 6. - Contrast of the reconstructed image vs. the position of the centre of the Gaussian AMTF with at fixed width, $w_{0}=400 \mathrm{l} / \mathrm{mm}$.

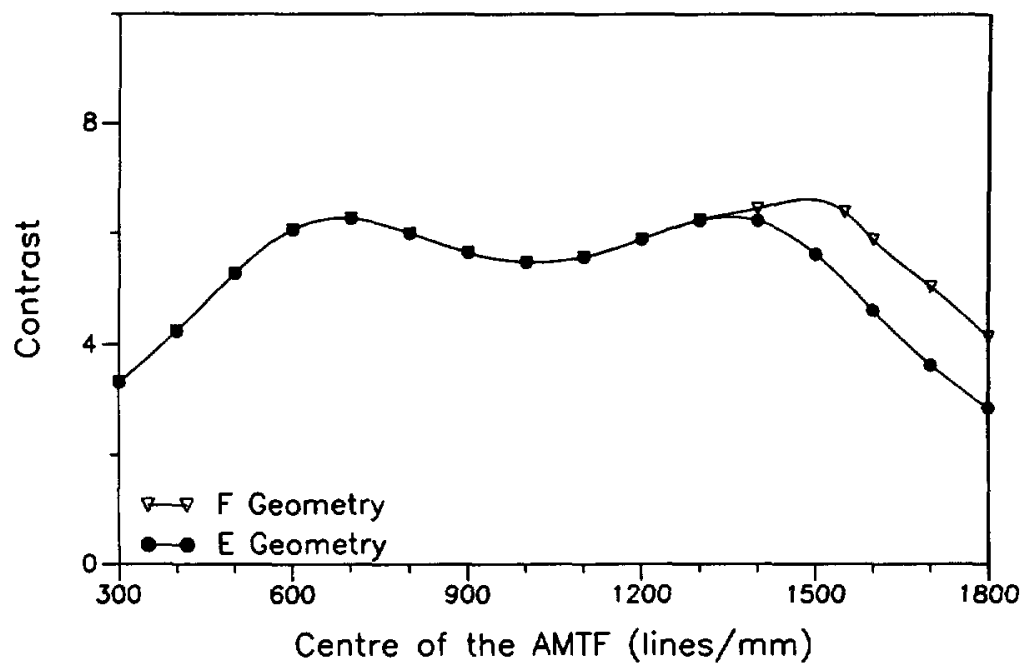

Fig. 7. - Contrast of the reconstructed image vs. the position of the centre of the Gaussian AMTF with at fixed width, $w_{0}=600 \mathrm{l} / \mathrm{mm}$.

AMTF should be «fine-tuned (e.g. by changing the thickness of the recording layer) in order to obtain optimal contrast. Figure 8 illustrates this comment by presenting the reconstructed images calculated in geometry $\mathrm{F}$ with the parameters corresponding to the two peaks and the dip in figure 6.

The thickness and other parameters of the photoconductor-thermoplastic devices can not be set easily in order to obtain the desired optimal amplitude modulation functions. However, the 

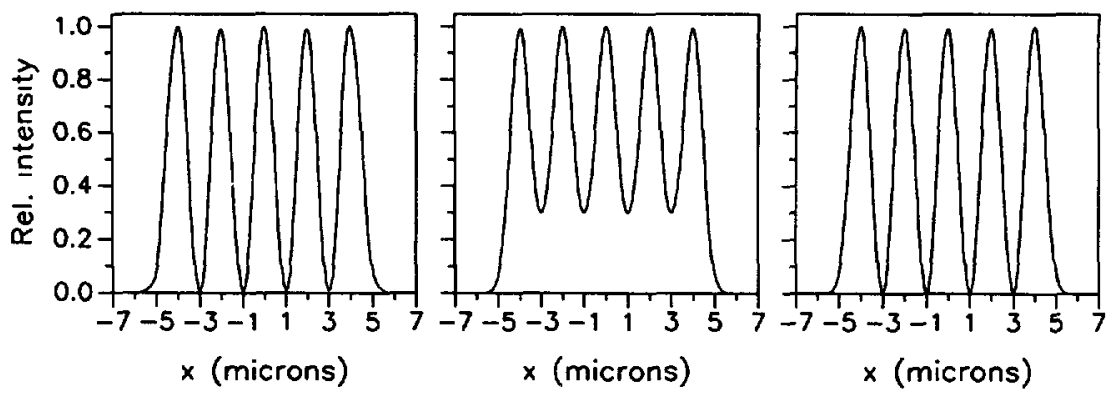

Fig. 8. - Intensity distribution of the reconstructed image in geometry $F$, with $w_{0}=400 \mathrm{l} / \mathrm{mm}$. Left : $\nu_{0}=800 \mathrm{l} / \mathrm{mm}$, middle : $\nu_{0}=1100 \mathrm{l} / \mathrm{mm}$, right : $\nu_{0}=1400 \mathrm{l} / \mathrm{mm}$.

angle of incidence of the reference wave can usually be changed more or less, depending on the optical and mechanical constraints of the setup. The contrast of the reconstructed image as a function of the reference wave angle (with AMTF parameters kept constant) has been calculated both in geometry $E$ (Fig. 9) and geometry F (Fig. 10). At $w_{0}=500 \mathrm{l} / \mathrm{mm}$ two pronounced maxima occur in both geometries ( $\nu_{0}$ remained constant for all the four curves).

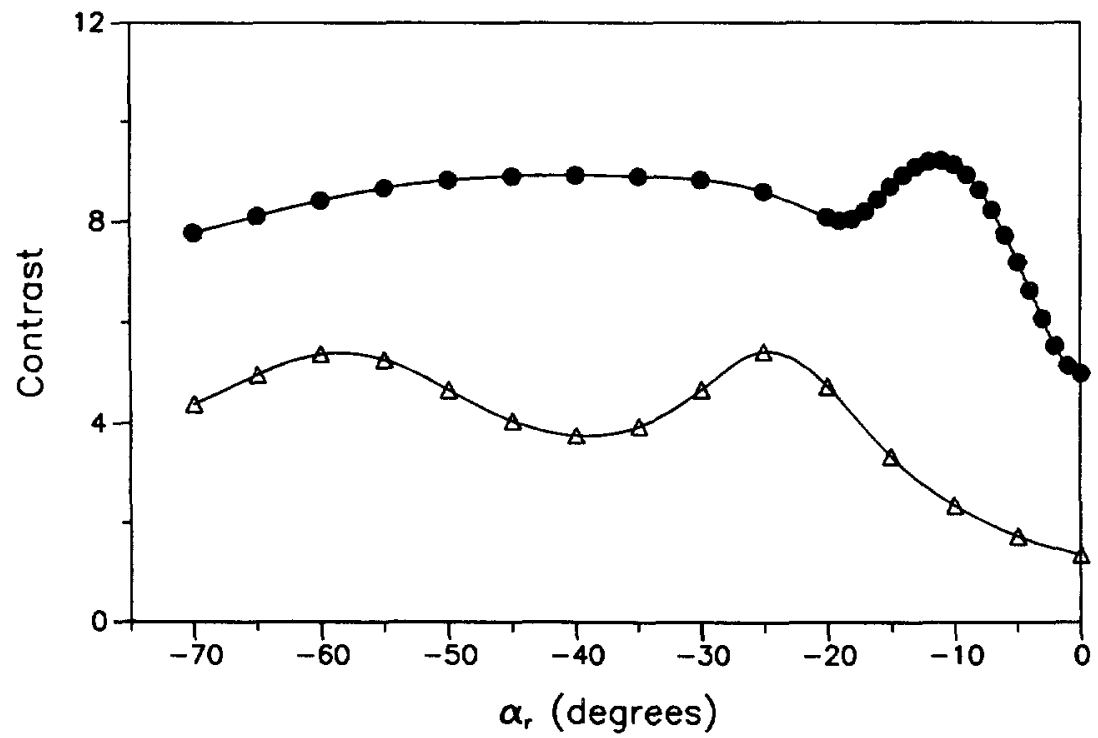

Fig. 9. - Contrast of the reconstructed image vs. the angle of incidence of the reference wave in geometry E. $\nu_{0}=1000 \mathrm{l} / \mathrm{mm}$. Upper curve : $w_{0}=1000 \mathrm{l} / \mathrm{mm}$, lower curve : $w_{0}=500 \mathrm{l} / \mathrm{mm}$.

Very interesting conclusions can be drawn from these double peaked curves. The local spatial frequency at the hologram centre corresponding to the first peak of the lower curve of figure 9 is $670 \mathrm{l} / \mathrm{mm}$ and that corresponding to the second peak is $1340 \mathrm{l} / \mathrm{mm}$. The dip between them corresponds to $1000 \mathrm{l} / \mathrm{mm}$ i.e. to $\nu_{0}$ (or in this special case to $2 w_{0}$ ), and it is the arithmetic mean value of the two former spatial frequencies. This also holds true for the lower curve of figure 10 if one takes into account the shift of the spatial frequency distribution of the hologram due to the off-axis position of the object. In geometry $E$ the curve corresponding to 


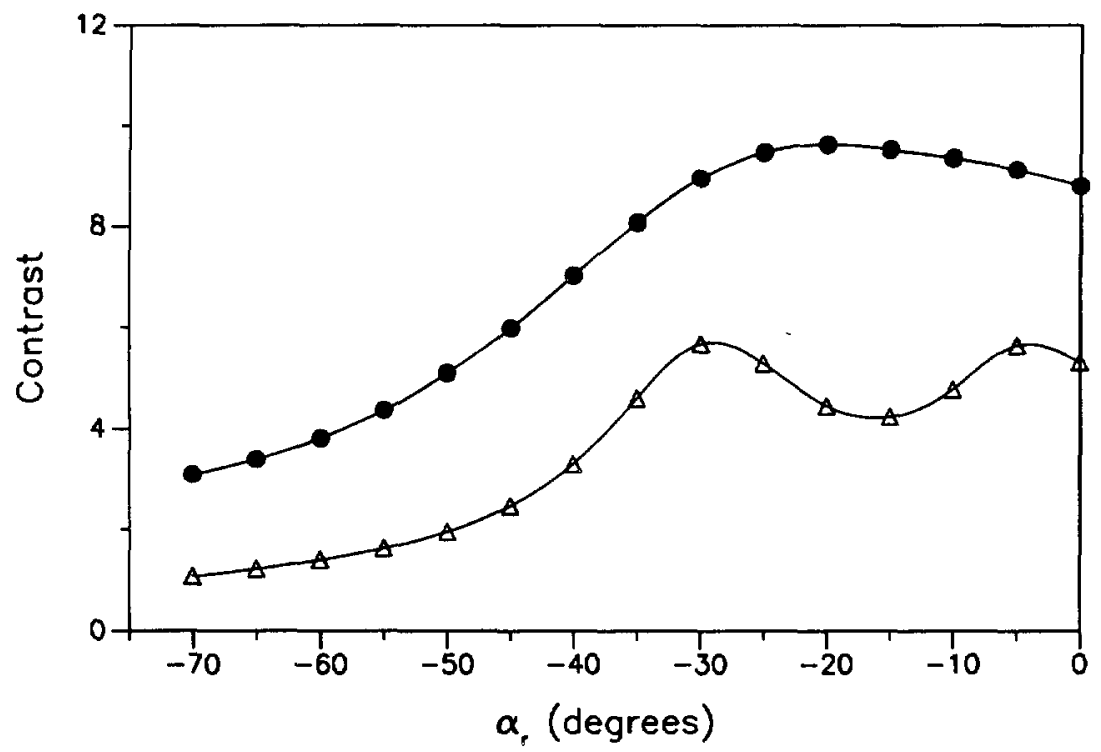

Fig. 10. - Contrast of the reconstructed image $v$ s. the angle of incidence of the reference wave in geometry F. $\nu_{0}=1000 \mathrm{l} / \mathrm{mm}$. Upper curve : $w_{0}=1000 \mathrm{l} / \mathrm{mm}$, lower curve $: w_{0}=500 \mathrm{l} / \mathrm{mm}$.
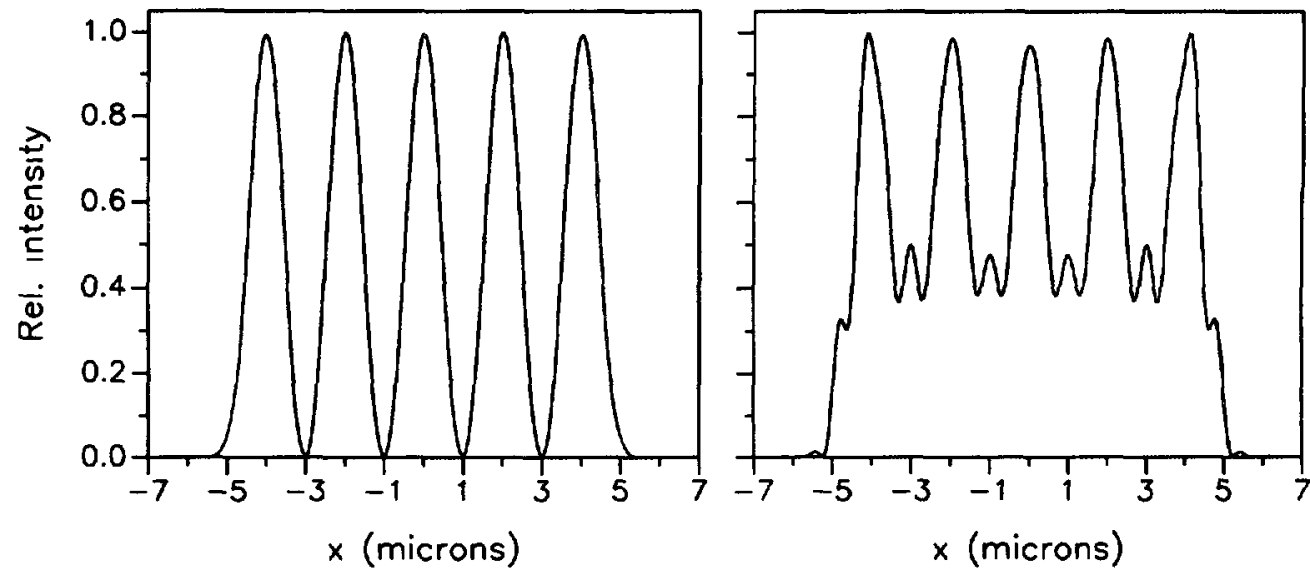

Fig. 11. - Intensity distribution of the reconstructed image in geometry $\mathrm{E}$, with $\nu_{0}=1000 \mathrm{1} / \mathrm{mm}$ and $w_{0}^{\prime}=500 \mathrm{l} / \mathrm{mm}$. Left : $\alpha_{\mathrm{r}}=-25$ degrees, right : $\alpha_{\mathrm{r}}=-10$ degrees.

$w_{0}=1000$ also has two maxima, though one is rather a plateau than a peak. The dip between them is at $\alpha_{\mathrm{r}}=18.5$ degrees corresponding to 500 lines $/ \mathrm{mm}$. The broad peak is at $\alpha_{\mathrm{r}}=40$ degrees $(1000 \mathrm{l} / \mathrm{mm})$. These interesting effects can readily be understood when one represents the intensity distribution of the object wave and the local diffraction efficiency due to the Gaussian AMTF as a function of the hologram coordinate in one graph. However, these discussions are beyond the scope of the present paper. Finally, a pair of images corresponding to the peak at 25 degrees of the lower curve of figure 9 and to the 10 degree point of the same curve, is presented. 


\section{Conclusions.}

A new method has been developed for the evaluation of the effects of the MTF of the thermoplastic recording materials on the reconstructed holographic image. The amplitude modulation transfer function of the thermoplastic materials have been modelled by Gaussian functions and inserted into the double diffraction integral. The capability of the method was demonstrated with several examples. Relationships between the contrast of the reconstructed image and the parameters of the AMTF of the recording material have been established. A nonmonotonic dependence of the image contrast on the position of the centre of the AMTF has been found, while a monotonic dependence on the width of the AMTF has been obtained.

One of the predictions of the present model is that it is possible to optimize the holographic recording for the actual type of thermoplastic material by a suitable choice of the recording geometry. For example in case of certain values of the AMTF parameters ( $\nu_{0}$ and $w_{0}$ ) there are two values of the reference angle which result in maximum image contrast.

Finally, the present calculations predict that in principle it is possible to retrieve high resolution images (down to $1 \mu \mathrm{m}$ at $\lambda=633 \mathrm{~nm}$ ) from thermoplastic holograms, too.

Experimental verification of the results of the present work seems to be straightforward, and in case of experimental proofs, this model may become a useful tool in the design of holographic optical elements.

\section{References}

[1] Glenn W. E., J. Appl. Phys. 30 (1959) 1870.

[2] Urbach J. C. and Meier R. W., Appl. Opt. 5 (1966) 666.

[3] Doyle R. J. and GlenN W. E., IEEE Trans. Electron Devices ED-18 (1971) 739.

[4] SHERIDON N. K., IEEE Trans. Electron Devices ED-19 (1972) 1003.

[5] Lin L. H. and Beauchamp H. L., Appl. Opt. 9 (1970) 2088.

[6] GaYNor J., IEEE Trans. Electron Devices ED-19 (1972) 512.

[7] Stewart W. C. et al., RCA Rev. 34 (March 1973) 3.

[8] Colbukin W. S. and Tompkins E. N., Appl. Opt. 13 (1974) 2934.

[9] Lo D. S., Johnson L. H. and Honebrink R. W., Appl. Opt. 14 (1975) 820.

[10] Meyrueis P., Liegeois C., Lamy F., Ineichen B., Large aperture thermoplastic film computer holographic recording system, Proceedings of SPIE, 353 (1983) p. 40.

[11] Cressman P. J., J. Appl. Phys. 34 (1963) 2327.

[12] Budd H. F., J. Appl. Phys. 36 (1965) 1613.

[13] Rabtchin A. I. et al., Zhu. Nautchn. Prikl. Fotogr. Kinematogr. 12 (1967) (in Russian) 149.

[14] Gruscho Yu. P., Ionkin P. A., Zhu. Nautchn. Prlkl. Fotogr. Kinematogr. 12 (1967) (in Russian) 166.

[15] Anderson H. R. Jr., Bartkus E. A., Reynolds J. A., IBM J. Research Development (March 1971) p. 140.

[16] Urbach J. C. and Meier W., Appl. Opt. 11 (1969) 2269.

[17] UrbaCH J. C., Thermoplastic Hologram Recording. Holographic Recording Materials, H. M. Smith Ed., Springer Topics in Applied Physics, 20 (Springer-Verlag, Berlin, Heidelberg, New York, 1977) p. 161.

[18] BÁNYÁSz I., KıSS G. and VARGA P., Holographic image of a point source in presence of misalignment, Appl. Opt. 27 (1988) 1293.

[19] BÁNYÁsz I., Resolution problems in holography, presented at Conference « DOE-91 » in Szlkarska Poreba, Poland, Proceedings of the SPIE, 1574 (1991) pp. 282-293.

[20] BÁNYÁSZ I., Resolution and field range limitations in holography imposed by film MTF and nonlinearity, Presented at the Conference Holographics'92 in London, U.K. (1992), Proceedings of SPIE 1732 (1993) 172-183.

[21] BÁNYÁsz I., Evaluation of the direct effect of the film MTF on the holographic image, J. Modern Opt. 40 (1993) 15. 